\title{
Alopecia Triangular congénita temporal en edad pediátrica. A propósito de un caso
} Temporary congenital triangular alopecia in pediatric age. About a case

Adrian Isacc Nieto Jiménez ${ }^{1}$, Yanel Benitez Sànchez ${ }^{2}$

DOI. 10.21931/RB/2020.05.02.10

Resumen: Se presenta el caso de una niña de 9 años de edad, valorada en el servicio de Dermatología provincial por ausencia de cabello presente desde el nacimiento. Se evalúa en conjunto y se concluye clínicamente como una Alopecia Triangular Congénita Temporal, sin necesidad de biopsia cutánea. Esta entidad es un trastorno de prevalencia desconocida, aunque quizá más frecuente de lo que podamos pensar, pero que en ocasiones puede constituir motivo de consulta al pediatra y/o al dermatólogo. Es una forma de Alopecia no cicatricial. Es necesario conocerla para evitar tratamientos y pruebas innecesarias para su diagnóstico por la gran similitud con otras dermatosis.

Palabras clave: Alopecia, congénita, dermatología.

Abstract: The case of a 9-year-old girl is presented, valued in the service of provincial Dermatology, by the absence of present hair from the birth. They were carried out several probable diagnoses, It is evaluated on the whole, and you conclude clinically like a Triangular Alopecia, without the necessity of cutaneous biopsy. This entity is a dysfunction of the unknown. However, the maybe more frequent prevalence of what we can think, but that on occasions it can constitute consultation reason to the pediatrician and the dermatologist. It is a form of non-cicatricial Alopecia. It is necessary to know it to avoid treatments and unnecessary tests for their diagnosis for the similarity with another skin disease.

Key words: Alopecia, congenital, dermatology.

\section{Introducción}

La Alopecia Triangular congénita, también llamada nevus de Brauer,es una forma de alopecia no cicatricial y circunscrita que fue descrita por primera vez por Sabouraud, dermatólogo francés, en el año $1905^{1}$.

Comenzó a tomar importancia cerca de 5 años después cuando se describen las causas de alopecia en el recién nacido, sobre todo la asociada a la sífilis congénita. Se trata de una condición benigna, no progresiva y muy poco conocida, probablemente más por infradiagnóstico y la escasez de publicaciones que por su frecuencia, se sitúa en torno al 0,11\% de las consultas de Dermatología en el mundo, más frecuente en la raza blanca, sin predilección de sexo, y se ha sugerido una herencia paradominante, que explica que sea de aparición esporádica; su patogenia es desconocida ${ }^{2,3}$.

Clínicamente consiste en una placa de alopecia circunscrita, permanente y asintomática, no se conoce el estímulo que produce la regresión folicular irreversible. La histopatología muestra unos folículos pilosebáceos hipoplàsicos sin alteraciones inflamatorias ni cicatriciales añadidas, siendo el número de folículos pilosos normal pero todos de carácter veUloso, con presencia de glándulas sebáceas normales en número y tamaño $0^{2,3,4}$.

El diagnóstico es fundamentalmente clínico, solo precisa de estudio histológico en casos de duda diagnóstica. Es importante referir el caso siempre al servicio de Dermatología Pediátrica para evitar exámenes y estudios innecesarios, en ocasiones invasivos para el niño $0^{3,5}$.

Se han descrito casos asociados a otros trastornos como síndrome de Daw, leuconiquia, facomatosis pigmento vascular, nevus de cabello lanoso, hiperpigmentaciòn sectorial del iris, retraso mental, epilepsia, malformación de Dandy-Walker , síndrome de Leopard, aplasia cutis congénita ${ }^{2,3}$, síndrome GLH( cráneo sinostosis, displasia cerebelosa, displasia trigeminal y retraso mental) y otros síndromes polimalformativos ( cardiopatía congénita y anormalidades renales y genitales), que posiblemente sean asociaciones causales ${ }^{6}$, aunque otros autores consideren que las alteraciones neurológicas del desarrollo y la facomatosis pigmento vascular no son simples asociaciones causales ${ }^{3}$.

El diagnóstico diferencial se realiza con otras formas de alopecia, que pueden observarse en la edad pediátrica ${ }^{5}$.

Esta dermatosis representa un reto diagnóstico para los dermatólogos, y casi siempre el paciente acude a la consulta con modificación de la piel alopécica o cicatrices por procederes invasivos ${ }^{4,6}$.

La tasa de incidencia en E.U no se precisa bien, aunque estudios reportan que representa el $1,4 \%$ de las consultas de dermatología en edad pediátrica, siendo más frecuente en el sexo femenino sobre el masculino, en proporción de 2:1. En Europa se han registrado formas clínicas de otra localización, como la frontal y occipital, asociadas a trastornos sistémicos del aparato genitourinario y malformaciones cardiovasculares ${ }^{6,7}$.

En Cuba, solo se ha reportado un caso de alopecia triangular congénita temporal en la Habana, se considera que se debe al subregistro en las consultas de otras especialidades o la rareza de la entidad en la Atención Primaria de Salud.

En Villa Clara no se recogen registros de esta dermatosis ${ }^{8}$.

En la actualidad no se dispone de un tratamiento efectivo. Por este motivo deberemos asegurarnos de explicarles bien en qué consiste esta entidad y en la ausencia de implicaciones

Especialista en primer grado en Medicina General Integral y Dermatología. Profesor Asistente. Diplomado en Hematodermias. Hospital Pediátrico Provincial:

José Luis Miranda. Santa Clara. Cuba.

${ }^{2}$ Especialista en primer grado en Medicina General Integral. Hospital Pediátrico Provincial: José Luis Miranda. Santa Clara. Cuba.

Corresponding author: adrianisacnj@gmail.com 
clínicas, para evitar actuaciones infructuosas o contraproducentes para la salud del paciente ${ }^{6}$.

\section{Caso clínico}

Se presenta la paciente YPR, femenina, de 9 años de edad, valorada en el servicio de Dermatología del Hospital Pediátrico Provincial José Luis Miranda, en Santa Clara, en septiembre del año 2019 por ausencia de cabello en un área del cuero cabelludo, presente desde el nacimiento. Había sido valorada anteriormente en otro centro de Salud con diagnóstico de alopecia areata y le indicaron varios tratamientos médicos con esteroides tópicos de mediana potencia en forma de masajes compresivos, sin mejoría clínica alguna; motivo por el cual acude a nuestro centro. Se procedió a examinarla e interrogarla en compañía de sus padres. Se revisa la literatura y se plantea como diagnóstico clínico una alopecia triangular congénita temporal.

\section{Examen Físico:}

Se observa área alopécica de aproximadamente $15 \mathrm{~cm}$ de diámetro, con bordes difusos y pequeños pelos alrededor, localizada en región frontotemporal derecha, de forma más o menos triangular, congénita, de superficie lisa, no presencia de escamas, ni atrofia, ni cambios de coloración, ni otro tipo de lesión en el momento del examen. (Figura 1 y 2)

\section{APP: Negativos}

APF: Madre con HTA con tratamiento con enalapril, $20 \mathrm{mg}$ diarios.

No se precisa de biopsia cutánea teniendo en cuenta el diagnóstico clínico de esta entidad.

\section{Se indican exámenes complementarios de rutina:}

Hemograma completo, eritro sedimentación, coagulograma completo, glicemia, perfil hepático y renal, LDH, VDRL, Antígeno de superficie para hepatitis B y VIH dentro de parámetros normales.

\section{Rx de tórax AP: normal}

Ya le habían realizado Rx de cráneo que fue normal.

Se lleva a discusión el caso en el servicio de Dermatología y se interconsulta con el servicio de Pediatría para descartar otros trastornos asociados, y se concluye como:

\section{Comentarios}

La Alopecia Triangular Congénita Temporal representa sin dudas una dermatosis en ocasiones de complejo diagnóstico por la similitud con otras entidades ${ }^{7,8}$.

El diagnóstico se basa en su naturaleza estable, su clínica característica, su localización y el aspecto normal de la piel afectada, no siendo necesario su estudio histológico.

Las manifestaciones clínicas de este cuadro son características y consisten en el hallazgo de una placa de alopecia circunscrita, no inflamatoria ni cicatricial, más habitualmente de forma unilateral que bilateral, como es el caso de nuestro paciente ${ }^{2-8}$

Su localización preferente es frontoparietal, aunque existen casos descritos en otras localizaciones ${ }^{3,8}$.

Se caracteriza por ser asintomática y permanente, de crecer lo haría en la misma proporción que el resto del cuero cabelludo 9 .

Puede estar presente desde el nacimiento o desarrollarse en los primeros meses de vida, haciéndose evidente habitualmente en los nueve primeros años de vida o, menos frecuentemente, en la adolescencia o en edad adulta ${ }^{3-9}$, por ello, en la actualidad se tiende a considerar adquirida más que congénita, teoría con la cual no están de acuerdo otros autores ${ }^{9,10}$.

La forma de la lesión es triangular con ángulos romos pero también ovalados o redonda ${ }^{2-5}$.

En el interior de la lesión se aprecia una piel normal con ausencia de pelos terminales y la existencia de pelos finos vellosos $^{5-10}$, que se pueden apreciar mejor con lupa9 .

En los bordes de la lesión puede haber pelos terminales raleados que se mezclan con el pelo del cuero cabelludo normal ${ }^{5}$.

El diagnóstico diferencial debe hacerse con otras causas de alopecia circunscrita: la alopecia areata (pilotracción+, presencia de pelos en signo de exclamación y zonas de piel con ausencia total de pelo); la aplasia cutis (ausencia total de pelo, piel deprimida y blanda al tacto y localización de elección en vértex); la alopecia cicatricial (abarca una amplia variedad de procesos como el lupus eritematoso cutáneo crónico, el liquen plano pilar o la alopecia mecánica por tracción, donde el resultado final es un cuero cabelludo brillante y duro al tacto, sin pelo o con cabellos en penacho); la presencia de un nevo sebáceo (tumoración amarillomarronácea); la tricotilomanía (presencia de cabellos de distintas longitudes y grosores variables, en ocasiones expresión de un conflicto emocional, alopecia de forma no triangular); la alopecia sutural; la alopecia vertical y la displasia focal bitemporal ${ }^{10}$.

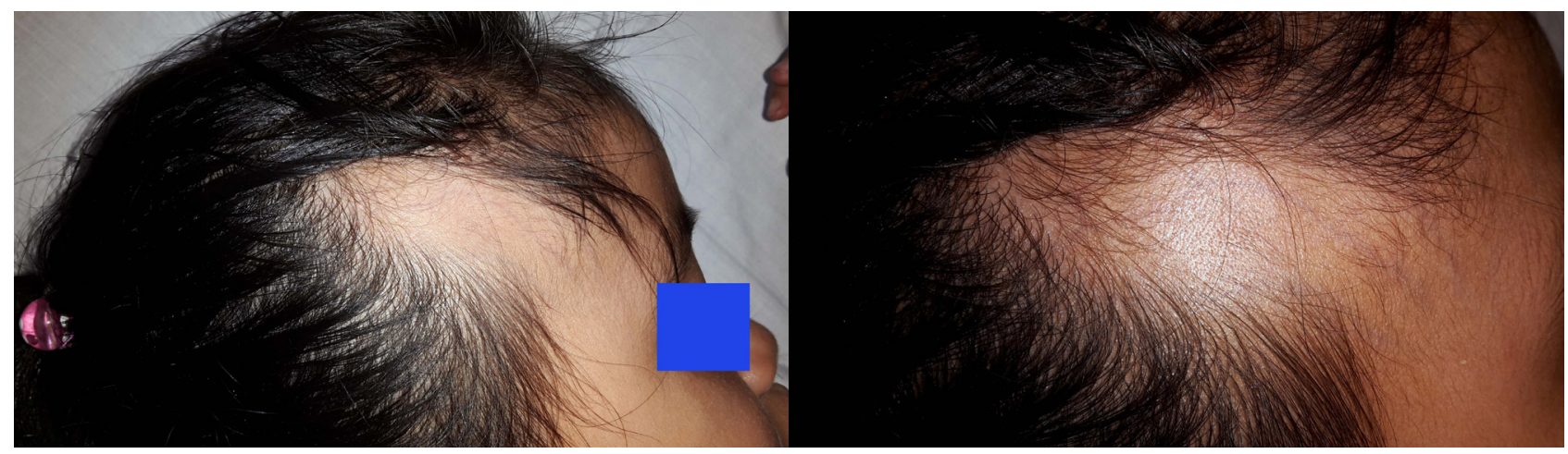

Figura 1 y 2. En ambas imágenes se observa el típico patrón de alopecia triangular temporal. En la figura 2 nótese que no existen cambios de textura ni color de la piel.

La alopecia se mantiene sin modificación hasta el momento. 
La Alopecia Triangular Congénita Temporal carece de tratamiento efectivo, que tampoco suele ser necesario. Se debe explicar la naturaleza de la entidad a los padres para que eviten tratamientos infructuosos o perjudiciales (sobre todo el uso de esteroides tópicos). El cuadro permanece estable de por vida, y tanto en varones como mujeres puede realizarse una extirpación quirúrgica del área afectada ${ }^{11}$.

Nuestro paciente muestra el patrón clínico y distribución característicos de esta dermatosis ${ }^{12}$.

\section{Conclusiones}

La Alopecia Triangular Temporal representa una entidad muy infrecuente pero cuyo reconocimiento es interesante para evitar tratamientos inútiles y con posibles efectos secundarios.

\section{Recomendaciones}

Esta dermatosis pertenece a las alopecias no cicatriciales de la infancia, trastorno subdiagnosticado en el mundo y en nuestro medio, motivo por el cual se recomienda que todo recién nacido con alopecia sea valorado por el servicio de Dermatología Pediátrica más cercano.

\section{Bibliographic references}

1. Saboraud R. Manuel. Elementaire de dermatology topographique regionale. Paris: Masson and Cie; 2014, p.197.

2. Garcìa -Hernàndez MJ, Rodrièguez Pichardo $A$, Camacho F. Congénita triangular alopecia (Brauer nevus) Pediatric Dermatol. 2014.

3. Leòn-Muiñòz E, Monteagudo $B$, Labandeira J, Cabanillas M. Alopecia triangular congénita bilateral asociada a cardiopatía congènita y anormalidades renales y genitales. Actas Dermosifilogr.2015; 99:573-82.

4. Lannicelli JC.Alopecia temporal triangular. Diagnóstico visual. Pediatría práctica (en línea) (actualizado el 27/12/2015; consultado el 19/03/2019.

5. Monteagudo B,Ginrte M,Leòn-Muiños E, Monteagudo JL, Toribio J. Alopecia triangular congènita. An pediatric (Barc). 2016,63(2): 175-84.

6. Sarifakioglu E, Esra Yilmaz A, Gorpelioglu C, Orun E. Prevalence of scalp disorders and hairs loss in children. Cutis. 2016, 90: 225-9.

7. Jiménez-Acosta F, Ponce I. Incidencia y prevalencia de las alopecias. Actas Dermosifilogr.2016; 100: 907:22.

8. Registro Estadístico de Salud Nacional. Cuba 2017.

9. Jiménez-Acosta F, Ponce I. Transplante de pelo en la alopecia temporal triangular. Actas Dermosifilogr. 2018; 28: 202-13.

10. Kudligi C, Vittal Bhagwat P, Shendre Eshwarrao M, Tandon N. Giant congenital triangular alopecia mimicking alopecia areata. Int J Trichology. 2018; 103(5): 367:87.

11. Elmer KB, George RM. Congenital triangular alopecia: Acase report and review. Cutis, 69(2019), pp.255-6.

12. Tosti A.Congenital triangular alopecia. Report of fourteen cases. J Am Acad Dermatol, 16 (2019), pp.991-3.

Received: 16 febrero 2020

Accepted: 5 marzo 2020 